\title{
Disseminação da Propriedade Intelectual como Estratégia para Políticas de Ciência, Tecnologia e Inovação: o caso do Sistema Pernambucano de Inovação (SPIn)
}

\author{
Dissemination of Intellectual Property as a Strategy for Science, \\ Technology and Innovation Policies: the case of the Pernambucano \\ Innovation System
}

André Marques Cavalcanti ${ }^{1}$

Eduardo Andrade Bemfica ${ }^{1}$

${ }^{1}$ Universidade Federal de Pernambuco, Recife, PE, Brasil

\begin{abstract}
Resumo
Este trabalho tem como objetivo propor ações de disseminação do conhecimento em Propriedade Intelectual (PI) para as instituições que compõem o Sistema Pernambucano de Inovação (SPIn) mapeadas no documento do governo do estado intitulado Estratégia de Ciência, Tecnologia e Inovação para Pernambuco 2017-2022 (ECT\&I$\mathrm{PE}$ ). A metodologia utilizada foi uma abordagem qualitativa, de caráter descritivo, baseada em análise da literatura correspondente ao tema e em pesquisa documental. No referencial teórico, houve uma exposição sobre o papel dos Sistemas Regionais de Inovação (SRI) e a importância da disseminação da Propriedade Intelectual (PI) como estratégia dentro do processo de inovação. Como resultado, são apresentadas propostas com linhas de ações de disseminação do conhecimento da PI agrupadas aos seis eixos estratégicos do documento, a fim de nortear e de impactar as políticas públicas de inovação para a área de Ciência, Tecnologia e Inovação do estado.
\end{abstract}

Palavras-chave: Desenvolvimento Tecnológico. Políticas Públicas. Sistema Regional de Inovação.

\begin{abstract}
This work aims to propose actions for dissemination of knowledge in Intellectual Property (IP) to the institutions that compose the Pernambuco Innovation System (SPIn) mapped in the state government document titled Science, Technology and Innovation Strategy for Pernambuco 2017-2022 (ECT \& I-PE). The methodology used was a qualitative approach, with a descriptive character, based on an analysis of the literature corresponding to the theme and on documentary research. In the theoretical framework, there was an exposition about the role of Regional Innovation Systems (SRI) and the importance of the dissemination of IP as a strategy within the innovation process. As a result, lines of IP knowledge dissemination actions grouped to the six strategic axes of the document are proposed, in order to guide and impact public innovation policies for the state's Science, Technology and Innovation area.
\end{abstract}

Keywords: Technological Development. Public Policy. Regional Innovation System.

Área Tecnológica: Educação. 


\section{Introdução}

O uso do sistema de Propriedade Intelectual (PI) é cada vez mais estratégico para a indústria do conhecimento. Segundo Gandelman (2004), a proteção do fruto intelectual estimula a criatividade e os investimentos em produção do conhecimento. Portanto, é essencial disseminar essa informação e capacitar os recursos humanos para utilizar a PI gerando riquezas e trazendo benefícios para a sociedade (TAKAGI; ALLMAN; SINJELA, 2008).

Segundo Sun e Baez (2009), a PI se configura como fundamental na educação devido às forças políticas, sociais e econômicas que fizeram do conhecimento e da pesquisa científica bens centrais na era da informação; sendo necessário que governo e empresas saibam fazer uso $e$ gerir o sistema de PI de forma técnica e atrelada aos interesses do mercado.

A interação e a cooperação entre as diversas organizações, sejam privados ou públicos, que fomentam a inovação de uma região são vitais para que todos se beneficiem com a difusão do conhecimento (FREEMAN, 1987; LUNDVALL, 1992; NELSON 1993). Segundo Freeman (1987) e Lundvall (1992), é preciso desenvolver relações profundas entre os agentes, estabelecendo um processo de integração e de cooperação de diferentes naturezas que envolvem não apenas empresas, mas diversos agentes, criando recursos humanos ativos que ajudem na construção do Sistema Nacional de Inovação (SNI).

Entre os conceitos do SNI, surge a abordagem sobre Sistemas Regionais de Inovação (SRI), que é a participação dos atores de suporte à inovação (COOKE: URANGA; ETXEBARRIA, 1997), sejam as universidades, institutos de C\&T públicos e privados, instituições do governo, habitats de inovação e agências de fomento que passam a trabalhar em conjunto com as empresas de uma região, tornando-as, assim, mais inovadoras e competitivas nacional e internacionalmente (DOLOREUX, 2002 ).

O fluxo de conhecimento é essencial para que uma região se torne inovadora (DOLOREUX; PARTO, 2005) e os atores presentes na região sejam importantes, porém é necessário compartilhar o conhecimento, porque, sem essa interação, a inovação poderá não acontecer. De acordo com Doloreux e Parto (2005), a utilização sistêmica de políticas públicas voltadas para o fluxo do conhecimento, que neste trabalho o foco é a PI, deve prevalecer para que todos os atores sintam-se parte do processo de inovação.

Na linha dos conceitos expostos, segundo Dubeux (2010), a PI e a inovação tecnológica foram consideradas eixos estratégicos centrais para o desenvolvimento socioeconômico dos países industrializados que primaram pela conquista de mercados por meio das exportações. Existe uma forte correlação entre o grau de desenvolvimento de um país e as leis e mecanismos de proteção à propriedade intelectual (SHERWOOD, 1992).

$\mathrm{Na}$ Coreia do Sul, por exemplo, país que passou pelo processo tardiamente semelhante de industrialização como o Brasil, na década de 1980, praticamente tinha o mesmo número de concessões de patentes de invenção por ano, como no Brasil (em torno de 2.300), sendo majoritariamente de invenções de estrangeiros (DUBEUX, 2010). Depois de várias políticas de CT\&I ao longo dos anos e de investimentos em educação de ensino básico e superior e em Pesquisa \& Desenvolvimento (P\&D) na indústria nacional (KIM, 2005), a Coreia do Sul tornou-se em 2016 o $4^{\circ}$ maior depositário de patentes do mundo com 216.694 pedidos de patentes; sendo $90 \%$ desses depósitos feitos por empresas coreanas, e o $11^{\circ}$ país mais inovador e competitivo 
entre 127 nações avaliadas pelo Índice Global de Inovação (ORGANIZAÇÃO MUNDIAL DA PROPRIEDADE INTELECTUAL, 2017a).

Entretanto, no Brasil, neste mesmo ano de 2016, houve 30.946 pedidos de patentes, colocando o escritório brasileiro de propriedade industrial - Instituto Nacional de Propriedade Industrial (INPI) - na $10^{a}$ posição no mundo em número de depósitos; contudo aproximadamente $20 \%$ desse total são feitos por residentes brasileiros, ou seja, mais de $80 \%$ das tecnologias protegidas no Brasil são de estrangeiros. Diante desse indicador e de outros, como despesas em educação e instrumentos de financiamento em $\mathrm{P} \& \mathrm{D}$, o Brasil estagnou-se na $69^{\circ}$ posição no Índice Global de Inovação (ORGANIZAÇÃO MUNDIAL DA PROPRIEDADE INTELECTUAL, 2017b).

O Estado de Pernambuco, apesar de ser destaque em algumas atividades científicas e tecnológicas, como o Parque Tecnológico Porto Digital, foi considerado o melhor do Brasil por três vezes (em 2007, 2011 e 2015) pela Associação Nacional de Entidades Promotoras de Empreendimentos Inovadores (ANPROTEC), no entanto, os números relacionados à proteção de propriedades industriais ainda são muito baixos.

Segundo o Boletim de Indicadores em CT\&I - Atividades de Patenteamento no país e no Estado de Pernambuco - período de 2000-2012 da Secretaria de Ciência, Tecnologia e Inovação de Pernambuco (SECTI-PE, 2015), os estados brasileiros com maior representatividade no total de concessões de patentes de invenção foram: São Paulo (52\%), Rio de Janeiro (13\%), Minas Gerias (10\%), Rio Grande de Sul (8\%), Santa Catarina (5\%) e Paraná (4\%). Esses mesmos estados foram os que obtiveram maior investimento em P\&D (MINISTÉRIO DA CIÊNCIA, TECNOLOGIA, INOVAÇÕES E COMUNICAÇÕES, 2014), os que possuem os maiores PIBs do Brasil (INSTITUTO BRASILEIRO DE GEOGRAFIA E ESTATÍSTICA, 2016) e também são os estados que mais depositaram patentes ao longo dos anos (INSTITUTO NACIONAL DE PROPRIEDADE INDUSTRIAL, 2016). Verifica-se, portanto, no Brasil, assim como nos países desenvolvidos, uma forte associação entre os investimentos feitos em P\&D, PIB e as concessões de patentes.

De acordo com o Boletim, o número de patentes de invenção concedidas a depositantes pernambucanos no período foi de apenas 22 , representando $0,51 \%$ das patentes brasileiras concedidas. No mesmo documento, para o período de 2006 a 2015, observa-se que 98\% dos pedidos de patentes de Pernambuco foram feitos por residentes de apenas 20 municípios pernambucanos, o que mostra a necessidade de disseminar o uso estratégico da PI aos demais atores do Sistema Pernambucano de Inovação (SPIn), a fim de promover a geração e a difusão desse conhecimento às empresas inovadoras e às cadeias produtivas do estado.

Em Pernambuco, foi lançada, em julho de 2017, a Estratégia de Ciência, Tecnologia e Inovação para Pernambuco 2017-2022 (ECT\&I-PE) que tem como macro-objetivo promover Ciência, Tecnologia \& Inovação (CT\&I) como valores para transformação social, elevação da qualidade de vida, da competitividade e da prosperidade, tudo baseado em conhecimento, aprendizagem e inovação (ECT\&I-PE, 2017).

De acordo com o ECT\&I-PE (2017), Pernambuco precisa estar preparado com indústrias mais competitivas e de alta produtividade e com a qualificação de sua força de trabalho voltada para as competências em Ciência, Tecnologia, Engenharia e Matemática.

A ausência do tema PI e do INPI como atores do SPIn nas linhas de ações da ECT\&I-PE configura-se como um problema para o desenvolvimento de um sistema de inovação moderno 
que contemple a transferência de tecnologia por meio de propriedades industriais como forma de agregação de valor e para a competição internacional. Dentro desse projeto desenhado para o Estado de Pernambuco não há ações estratégicas para a disseminação do conhecimento da importância da PI e para definir como funciona o sistema de patentes no Brasil, principalmente sobre a exclusividade temporária de direito ou a recompensa conferida ao inventor pela invenção (BARBOSA, 2010).

Portanto, o objeto deste estudo é analisar o documento Estratégia de Ciência, Tecnologia e Inovação para Pernambuco 2017-2022 (ECT\&I-PE), no qual se encontram mapeadas as instituições do SPIn e, portanto, pretende-se propor e investigar as linhas de ações de disseminação do conhecimento em PI, a fim de impactar e de fortalecer a inovação e garantir competitividade ao sistema com os mercados nacional e internacional.

\section{Sistema Regional de Inovação}

Um dos primeiros a conceituar os Sistemas Regionais de Inovação (SRI) foi o inglês Philip Cooke em 1992. Segundo Cooke, Uranga e Etxebarria (1997), o SRI é um sistema em que as empresas e outras organizações estão envolvidas de forma sistemática com uma aprendizagem interativa por meio de um ambiente institucional regional bastante enraizado. Um dos principais argumentos para a identificação do SRI é que as diversas regiões que fazem parte de um país possuem suas próprias características históricas, culturais, sociais, econômicas e políticas, criando identidades próprias, tornando-se diferentes umas das outras com seus próprios sistemas de inovação (COOKE; URANGA; ETXEBARRIA, 1997).

Para Cooke, Uranga e Etxebarria (1997), a inovação implica mudança nas organizações, nas instituições e no comportamento dos atores, em como eles se comunicam. Contudo, mudanças institucionais são um processo lento e demandam um período de tempo longo para criar uma certa maturidade. Para os autores, em um sistema dinâmico, o conhecimento precisa circular entre todos de forma interativa, a fim de multiplicar a informação. A forma como uma organização se constitui, como lida com o setor público e como os sistemas de ciência e tecnologia e $\mathrm{P} \& \mathrm{D}$ são organizados define um Sistema de Inovação (SI).

De acordo com Doloreux e Parto (2005), a inovação ocorre em um contexto regional no qual prevalecem regras, convenções e normas que surgem por meio de fatores econômicos e socioculturais que especificam o desenvolvimento tecnológico e econômico de cada região. Segundo Doloreux (2002), o conjunto de atores públicos e privados de uma região é capaz de aumentar e de melhorar a capacidade de aprendizado de uma localidade. Para o autor, o conjunto de atores de um SRI encoraja as organizações e outras empresas de uma região a desenvolverem formas específicas de capital que derivam das relações sociais, de normas, de valores e de interações de dentro da comunidade. Para o autor, tais características institucionais da região, infraestrutura e sistemas de transferência de conhecimento representam condições essenciais para estimular e promover atividades de inovação (DOLOREUX, 2002).

Segundo Lima, Ferreira e Fernandes (2007), em um SRI, a cooperação entre firmas, a criação de novos conhecimentos e a difusão via organizações, como universidades, instituições de ensino e de P\&D, de transferência tecnológica, geram uma cultura e ambiente propícios à inovação. Ou seja, para os autores, a interação entre agentes se transforma numa prática 
obrigatória. Contudo, segundo os mesmos autores, a força do sistema de aprendizagem local depende de um crescente número de fatores intangíveis, o que inclui a dinâmica da região, de bens socioculturais e políticos, do fluxo informal de informação e o conhecimento entre diferentes partes (LIMA: FERREIRA; FERNANDES, 2007).

Segundo Cooke (2001), a organização da estrutura de apoio à inovação ocorre de forma horizontal, dentro da região e, de forma vertical, entre regiões, estados, países e blocos econômicos. Dessa forma, há diferentes abordagens e configurações do suporte organizacional à inovação regional.

Segundo Autio (1998), a estrutura de SRI é dividida em dois subsistemas que caracterizam a principal construção desse sistema:

a)Subsistema de aplicação e exploração do conhecimento - constituído pelas empresas privadas, o mercado.

b)Subsistema de geração e difusão do conhecimento - constituído por instituições que participam na produção e na disseminação do conhecimento tecnológico tácito e nas habilidades técnicas, como universidades, centros de PD\&I, institutos de CT\&I, etc.

Para o SRI ocorrer de forma satisfatória, tais subsistemas são influenciados pelas organizações do SNI com suas políticas e instrumentos; outros SRIs; organizações internacionais; e instrumentos de políticas internacionais que influenciam diretamente a estrutura de SRI.

\section{Disseminação da Propriedade Intelectual}

Segundo a Organização Mundial da Propriedade Intelectual (2011), entende-se que a disseminação da cultura de PI deve ocorrer para haver um sistema de inovação estruturado com os seus atores atuando em conjunto para se desenvolver uma economia sustentável. Para essa entidade, a inovação é o condutor central do crescimento econômico, desenvolvimento e melhores empregos. É a principal ferramenta estratégica que permite que as empresas possam competir no mercado global e cria o processo pelo qual as soluções são encontradas para o desenvolvimento social e os desafios econômicos (ORGANIZAÇÃO MUNDIAL DA PROPRIEDADE INTELECTUAL, 2011).

Com a adoção do Acordo TRIPS, em 1994, vários países começaram a implantar diferentes programas para disseminar o conhecimento da propriedade intelectual, pois se sabia da falta de conhecimento e expertise sobre PI na maioria dos países à época das negociações do acordo TRIPS (DRAHOS, 1995) e se percebeu a importância que a PI teria na elaboração de políticas públicas voltadas para inovação e, consequentemente, atingindo as empresas (TAKAGI; ALLMAN; SINJELA, 2008). Drahos (1995) relata que nos anos de 1980, por exemplo, estimava-se que $40 \%$ do total de ativos das empresas privadas dos Estados Unidos da América eram compostos de ativos intangíveis. Atualmente, o percentual aumentou para aproximadamente mais de $70 \%$.

Segundo Takagi, Allman e Sinjela (2008), já havia discussões nacionais e internacionais sobre o tema PI há bastante tempo, contudo o treinamento e a discussão estavam atrelados há um modelo tradicional voltado, na maioria das vezes, apenas para advogados de grandes 
empresas. Com a demanda crescente por profissionais especializados em PI, após o acordo TRIPS não se conseguiu atender às demandas do mercado.

Em 1998, a OMPI, com o objetivo de atender à nova necessidade por conhecimento em PI gerada por TRIPS, criou a primeira Academia em PI do mundo (MENDES; BORHER, 2012). Nesse momento, a OMPI assumiu papel fundamental junto aos países da Organização Mundial de Comércio (OMC) com programas de capacitação de recursos humanos, assistência legislativa, promoção de atividades de sensibilização e outros serviços e programas desenvolvidos de acordo com as necessidades dos países-membros.

Segundo Mendes e Borher (2012), a partir de então mais de 20 países já criaram suas próprias Academias de PI (API), a grande maioria vinculada aos escritórios nacionais que garantem a proteção dos ativos intangíveis. Tais Academias oferecem treinamento profissional especializado, cursos de capacitação para gestores de curto, médio e longo prazos, inclusive cursos de pós-graduação, além de pesquisas para aprimorar o conhecimento na área. Com a formação das APIs, foi criado a Rede Global de Academias de Propriedade Intelectual (GNIPA, sigla em inglês), que tem como objetivo principal promover uma cultura do uso do sistema de PI de forma integrada para os seguintes públicos-alvo: pesquisadores/inventores; gestores de empresas e profissionais de PI; formuladores de políticas públicas; funcionários de entidades governamentais; e estudantes e membros da sociedade civil em geral (MENDES; BORHER, 2012).

Para Amorim-Borher (2008), há uma necessidade na formação de recursos humanos para atuarem na gestão do conhecimento dentro das instituições de ciência e tecnologia, das agências de fomento e de Centros de P\&D das empresas e universidades. A formação multidisciplinar nessa área passa a ser essencial e importante para os governos e para os demais atores do Sistema Nacional Inovação (SNI) (MENDES; BORHER, 2012), criando de forma estratégica capacitações de profissionais que atendam às políticas nacionais de inovação e industrial.

Diante disso, segundo Takagi, Allman e Sinjela (2008), são muitos os desafios da educação de PI em vários países, contudo, por meio de aportes financeiros atrelados a políticas públicas expressivas nos campos industrial e de CT\&I, consegue-se capacitar as universidades para formarem massa crítica na área de PI. Conseguir implantar a PI como disciplina obrigatória na graduação e na pós-graduação contribui para transformar esse conhecimento dentro das universidades e, consequentemente, com os atores envolvidos nos SNIs.

No Brasil, as atividades de disseminação em PI vêm sendo promovidas por meio de duas grandes vertentes: as de curto prazo e as de pós-graduação (AMORIM et al., 2007). As de curto prazo são realizadas tradicionalmente por entidades de classes - Associação Brasileira da Propriedade Intelectual (ABPI), Associação Brasileira dos Agentes da Propriedade Industrial (ABAPI), etc. - e por instituições interessadas em debater o tema - Secretarias Estaduais, Sebraes, CNIs, etc. - por meio de eventos, congressos e de seminários.

O INPI, a partir de 2005, em parceria com atores do SNI, passou a promover uma série de treinamentos para gestores de tecnologia de 40 a 64 horas sobre os conceitos de PI. Em 2012, o Instituto criou um curso de ensino a distância sobre PI, o DL101PBR, em parceria com a OMPI que possibilitou uma capilaridade maior de atuação pelo Brasil, possuindo a partir de 2016 quatro edições anuais com 900 pessoas em média sendo certificadas por cada edição.

De acordo com Guimarães (2013), a disseminação de ensino em PI na pós-graduação surgiu inicialmente no Brasil em 2001 no CEFET-RJ em parceria com o INPI. Foram três edições formando servidores do INPI e de outras instituições. Segundo a autora, a partir de 2004, 
algumas especializações na área foram criadas pelo país, porém são poucas as instituições que mantiveram os seus programas, como é o caso da Unicamp e da PUC/Rio.

Atualmente, o Fórum Nacional de Gestores de Inovação e Transferência e Tecnologia (FORTEC), por meio do seu Programa de Mestrado Profissional em Propriedade Intelectual e Transferência de Tecnologia (PROFNIT), iniciado em 2016, também, é uma experiência em andamento, pois está inserido em mais de 14 estados brasileiros com diversos pontos focais e a intenção de formar já em 2018 mais de 100 profissionais para atuar na área de PI e transferência de tecnologia pelo país. Foi aberto, no ano de 2018, um novo edital que contemplará mais 145 vagas para novos profissionais em 10 novos estados do Brasil.

Em Pernambuco, apenas a partir do ano de 2011, iniciou-se um trabalho mais contínuo quando foi firmado um Acordo de Cooperação Técnica (ACT) de capacitação de PI entre o INPI, a Universidade Federal de Pernambuco (UFPE), o Sebrae/PE e a Secretaria de Ciência, Tecnologia e Inovação de Pernambuco, antiga Sectec, com duração de dois anos, que implicou uma nova dinâmica de disseminação no estado a partir deste ano.

Observou-se um salto considerável no número de depósitos de patentes logo no primeiro ano de implantação da nova gestão focada em disseminação comparando com o ano de 2010. De acordo com a Tabela 1, é possível observar que Pernambuco passou da $12^{a}$ posição em 2010 para a $7^{a}$ posição em 2017 em relação aos demais estados brasileiros. Somando o total dos últimos oito anos, está na 9a posição. Portanto, a partir de 2011, várias instituições - Agências de Desenvolvimento do Estado (AdDiper), Sebrae, IEL, Federação da Indústrias, órgãos de classe, como a OAB, secretarias e etc. - passaram a firmar parcerias com o INPI local, promovendo o conhecimento da PI por meio de seminários, palestras, cursos e workshops em universidades, instituições de ciência e tecnologia, escolas técnicas, empresas, parques tecnológicos e demais atores do sistema local de inovação.

Tabela 1 - Depósitos de pedido de patentes - período 2010 a 2017

\begin{tabular}{lccccccccr}
\hline Estado & $\mathbf{2 0 1 0}$ & $\mathbf{2 0 1 1}$ & $\mathbf{2 0 1 2}$ & $\mathbf{2 0 1 3}$ & $\mathbf{2 0 1 4}$ & $\mathbf{2 0 1 5}$ & $\mathbf{2 0 1 6}$ & $\mathbf{2 0 1 7}$ & TOTAL \\
1 São Paulo & 2933 & 3256 & 3224 & 3.153 & 2.935 & 2.773 & 2.696 & 2.659 & 23629 \\
2 Rio Grande do Sul & 711 & 788 & 811 & 859 & 737 & 695 & 835 & 759 & 6195 \\
3 Minas Gerais & 696 & 680 & 718 & 734 & 710 & 728 & 826 & 953 & 6045 \\
4 Paraná & 643 & 661 & 678 & 800 & 670 & 655 & 756 & 761 & 5624 \\
5 Rio de Janeiro & 519 & 577 & 553 & 572 & 584 & 538 & 870 & 858 & 5071 \\
6 Santa Catarina & 651 & 583 & 522 & 548 & 510 & 570 & 575 & 595 & 4554 \\
7 Bahia & 169 & 197 & 192 & 178 & 154 & 160 & 163 & 173 & 1386 \\
8 Espírito Santo & 113 & 130 & 135 & 170 & 154 & 196 & 195 & 151 & 1244 \\
9 Pernambuco & $\mathbf{6 7}$ & $\mathbf{1 0 8}$ & $\mathbf{9 3}$ & $\mathbf{1 1 2}$ & $\mathbf{1 4 3}$ & $\mathbf{1 7 5}$ & $\mathbf{1 9 0}$ & $\mathbf{2 2 3}$ & $\mathbf{1 1 1 1}$ \\
10 Goiás & 105 & 147 & 145 & 127 & 131 & 140 & 139 & 171 & 1105 \\
11 Distrito Federal & 114 & 143 & 143 & 145 & 123 & 113 & 130 & 152 & 1063 \\
12 Ceará & 104 & 93 & 82 & 117 & 115 & 122 & 153 & 187 & 973 \\
13 Paraíba & 47 & 34 & 56 & 52 & 32 & 53 & 80 & 203 & 557 \\
14 Rio Grande do Norte & 24 & 32 & 55 & 60 & 25 & 77 & 57 & 69 & 399 \\
15 Amazonas & 50 & 55 & 42 & 52 & 39 & 33 & 38 & 40 & 349 \\
\hline
\end{tabular}

Fonte: Instituto Nacional de Propriedade Intelectual (2018) 


\section{Metodologia}

A metodologia utilizada foi uma abordagem qualitativa, de caráter descritivo, baseado em análise da literatura correspondente ao tema e em pesquisa documental sobre o caso estudado. Tal pesquisa teve como principais fontes de consulta: boletins estatísticos de PI e documentos de CT\&I do Brasil e do mundo com a finalidade de adaptar as boas práticas.

De acordo com Cooke (2005), existem dois subsistemas-chave que precisam existir para que qualquer SRI funcione: 1) Subsistema de aplicação e exploração de conhecimento que são as empresas que usam o conhecimento para obter retorno comercial e; 2) Subsistema de geração e difusão de conhecimento que é composto, principalmente, de organizações públicas, como: institutos de pesquisa, agências de fomento e transferência de tecnologia, universidades e outros órgãos responsáveis pelas políticas públicas de inovação.

A proposta será, portanto, apresentada por meio da análise dos objetivos e linhas de ações dos seis eixos estratégicos descritos no documento da ECT\&I-PE, direcionada às organizações que fazem parte do Subsistema de geração e difusão do conhecimento do SPIn para melhor otimizar as ações de disseminação da PI para atender a esse SRI.

No intuito de facilitar o entendimento e a aplicação das ações sobre as diversas organizações que compõem o SPIn e as diferentes atividades prestadas por esses atores, propôs-se a utilização da classificação utilizada por Sousa Júnior (2014) em seu trabalho de mestrado, que agrupa as organizações do Subsistema de geração e de difusão do conhecimento proposta por Cooke em seis dimensões de atuação:

a) Científica - organizações voltadas à geração do conhecimento.

b) Tecnológica - organizações voltadas à realização de pesquisa aplicada.

c) Intermediação - organizações que buscam facilitar o processo de interação entre empresas e centros do conhecimento.

d) Capacitação e Gestão Empresarial - organizações que atuam na formação de executivos e profissionais para melhoria da gestão empresarial.

e) Financiamento - organizações que possuem linhas de fomento e/ou financiamento à inovação.

f) Governança - organizações que atuam na elaboração e gestão de políticas de apoio à inovação.

Essa divisão, demonstrada na Tabela 2, permitirá que as linhas de ações de disseminação da PI propostas fiquem distribuídas de forma mais harmoniosa entre as organizações e, assim, é possível obter um maior alcance entre elas. 
Tabela 2 - Dimensões do SPIn - Organizações separadas por dimensão

\section{DiMENSÕES}

1) Científica - geração de conhecimento

2) Tecnológica - Pesquisa Aplicada

3) Intermediação - Interação entre empresas, centros de conhecimento e demais agentes do SRI

4) Capacitação e Gestão empresarial

5) Financeira

6) Governança - Elaboração e gestão de políticas

Total

\section{ORganizaçõES Do SPIN}

7 Universidades e Institutos de Ensino

6 Laboratórios de Pesquisa e

Serviços Tecnológicos

5 Habitats de inovação

5 Empresas públicas do estado

6 Institutos Nacionais de

Ciência e Tecnologia

10 Centros Privados de Inovação

11 Incubadoras e aceleradoras

11 Institutos e empresas

públicas de pesquisa

6 Organizações de

Representação empresarial

2 Agências de Fomento

2 Ambientes regulatórios

Fonte: Adaptada de Sousa Júnior (2014)

Em contraponto, de acordo com o seu macro-objetivo, a ECT\&I-PE divide-se em seis eixos estratégicos centrais, já que cada um dos eixos é composto de um objetivo principal com linhas de ações para cada um deles, conforme descritos na Tabela 3:

Tabela 3 - Eixos estratégicos da ECT\&I-PE

\section{Eixo EstratéGico}

Eixo Estratégico 1:

Desenvolvimento de talentos e criatividade

Eixo Estratégico 2:

Pervasiva expansão da economia e sociedade digitais

Eixo Estratégico 3:

Aceleração da inovação nas atividades econômicas

Eixo Estratégico 4:

Cooperação e transferência de conhecimento

Eixo Estratégico 5:

Ambiente favorável à inovação

Eixo Estratégico 6:

Governança e responsabilidade

\section{Objetivo Principal}

Aumentar competências e capacidades para absorção, produção e difusão de tecnologias.

Preparar a sociedade e a economia para as novas bases produtivas com uso intensivo de TICs.

Ampliar a geração de valor e as competências inovativas.

\section{Ampliar a capacidade e otimizar tempo,} recursos e resultados para inovação.

Aprimorar os instrumentos e instituições para ampliar a capacidade inovativa.

Calibrar a política pública e despertar compromisso na sociedade por CT\&I.

Fonte: ECT\&I (2017) 
A partir dos seis eixos estratégicos da ECT\&I-PE, foi feita uma associação com as organizações do SPIn separadas nas seis dimensões apresentadas para, assim, propor linhas de ações de disseminação do conhecimento em PI que se articule de forma estratégica a todos os atores desse SRI.

\section{Resultados e Discussão}

O trabalho resultou na identificação dos principais atores do SPIn listados no documento da ECT\&I-PE 2017-2022 e separou por eixo estratégico por meio das dimensões de Sousa Júnior (2014) as linhas de ações que poderiam ser impactadas ao SRI estudado, a fim de criar um ambiente propicio ao uso estratégico da PI a longo prazo.

Foram elaborados três quadros, divididos por dois eixos estratégicos cada, para se discutir formas de disseminações da PI dentro da atual realidade do SPIn. Pela pesquisa em documentos de políticas de CT\&I realizada no Brasil e no mundo (Japão e Coreia do Sul, por exemplo) foram apresentadas propostas de disseminação do conhecimento em PI para os atores do SPIn.

No Quadro 1, tem-se, no Eixo Estratégico 1: Desenvolvimento de talentos e criatividade, as ações mais importantes que poderiam surtir um efeito imediato junto aos desenvolvedores e produtores de tecnologias, e, ao mesmo tempo, a longo prazo, pois se sugere a disseminação para os jovens de escolas técnicas do estado, assim como promovido no Japão. Segundo Ogiya (2016), no Japão existem diversas ações voltadas para a educação no ensino médio, como: o Clube da Invenção para Meninos e Meninas com mais de 9.000 membros e 2.000 professores pelo Japão discutindo inovação, empreendedorismo e PI; Feiras de ciências e tecnologia para alunos do ensino médio; Publicação das invenções em revistas; Premiações Nacional e Regional de Melhor Invenção; Campanha contra pirataria; Seminários de PI; Dia do Jovem Inventor; Premiações; e Desenvolvimento de material didático próprio para jovens.

Assim como no Japão, na Coreia do Sul, segundo Cassiolato e Zucoloto (2014), o grau de desenvolvimento tecnológico das empresas locais e a promoção da educação e infraestrutura em CT\&I foram alguns dos aspectos essenciais para o sucesso econômico e industrial do país sul coreano.

No Eixo Estratégico 2, o Instituto Senai de Pernambuco será referência na área de TI entre todos do pais e deve capacitar seus professores para estarem atentos às invenções passíveis de proteção que irão desenvolver junto aos seus alunos, com o Porto Digital, devem ter um trabalho mais próximo do INPI, como um Acordo de Cooperação Técnica entre as instituições, para melhor atender à demanda local na área de TICs. 
Quadro 1 - Eixos Estratégicos 1 e 2: Proposta linhas de ações para o Sistema Pernambucano de Inovação (SPIn)

\begin{tabular}{|c|c|c|}
\hline Eixo ESTRATÉGICO E OBJETIVO & Dimensão & LiNHAS DE AçÕES \\
\hline $\begin{array}{c}\text { Eixo Estratégico 1: } \\
\text { Desenvolvimento de } \\
\text { talentos e criatividade } \\
\text { Objetivo: aumentar } \\
\text { competências e capacidades } \\
\text { para absorção, produção } \\
\text { e difusão de tecnologias. }\end{array}$ & $\begin{array}{c}\text { Científica } \\
\text { Geração de } \\
\text { conhecimento }\end{array}$ & $\begin{array}{l}\text { - Capacitar professores das redes de ensino pública (escolas técnicas e } \\
\text { universidades) em conhecimento da PI de diferentes níveis das áreas de } \\
\text { Ciência, Tecnologia, Engenharias, Matemática e Computação. } \\
\text { - Formar em nível avançado pessoal qualificado como ponto focal dentro de } \\
\text { cada um dos Centros Privados de Inovação, Incubadoras e Aceleradoras, } \\
\text { Laboratórios de Pesquisa e Serviços Tecnológicos, Institutos e empresas } \\
\text { Públicas de pesquisa e ICTs do estado. } \\
\text { - Desenvolver material didático com linguagem adequada sobre PI específica } \\
\text { para alunos do ensino fundamental e médio da rede pública do estado. } \\
\text { - Criar premiações estaduais de tecnologias estratégicas para o estado com } \\
\text { apoio na escrita, busca patentária e depósito do pedido de patente. } \\
\text { - Ampliar o projeto "Consórcio de Universidades" da OMPI/INPI na utilização } \\
\text { do curso à distância DL101PBR como atividade complementar para todas } \\
\text { as universidades pernambucanas públicas e privadas. }\end{array}$ \\
\hline $\begin{array}{l}\text { Eixo Estratégico 2: } \\
\text { Pervasiva expansão } \\
\text { da economia e } \\
\text { sociedade digitais. } \\
\text { Objetivo: preparar a sociedade } \\
\text { e a economia para as novas } \\
\text { bases produtivas com } \\
\text { uso intensivo de TICs. }\end{array}$ & $\begin{array}{c}\text { Tecnológica } \\
\text { Pesquisa } \\
\text { Aplicada }\end{array}$ & $\begin{array}{l}\text { - Capacitar alunos e professores do Instituto Senai de Inovação - Tecnologia } \\
\text { da Informação de PE com atividades de PI e premiação de invenções } \\
\text { patenteadas na área de TIC. } \\
\text { - Prospectar e monitorar por meio de bancos de patentes, as tecnologias } \\
\text { na área de inteligência artificial, sistemas embarcados, robótica, Big Data, } \\
\text { computação em nuvem. } \\
\text { - Inserir o Senai/PE no ACT em tramitação entre o INPI e o Porto Digital para } \\
\text { disseminar o uso estratégico da PI para as empresas embarcadas e startups } \\
\text { nestes parques tecnológicos. }\end{array}$ \\
\hline
\end{tabular}

Fonte: Elaborado pelos autores deste artigo (2018)

No Quadro 2, os Eixos Estratégicos 3 e 4 são voltados para a interação entre empresas, centros de conhecimento e demais agentes do SRI e na capacitação e gestão empresarial. Segundo Chang (2002), o sucesso das políticas industriais dos países asiáticos (Japão, Coreia do Sul, Índia e China) foi galgado utilizando diversas ações com objetivo de proteger o setor industrial, como restrições às importações, investimento de setores estratégicos e em capital humano e criação e promoção de conglomerados econômicos.

Na Coreia do Sul, o uso estratégico da PI foi introduzido por meio do bem-sucedido processo de política industrial denominado "aprendizado tecnológico", quando o país intensificou as atividades de $\mathrm{P} \& \mathrm{D}$, a fim de fortalecer sua competitividade internacional por meio da imitação criativa de sofisticadas tecnologias de outros países (KIM, 2005). Ainda segundo o autor, a partir daí, as empresas foram capacitadas a desenvolverem novas tecnologias por meio da observação e da utilização de tecnologias já existentes de acordo com ramos industriais em grande desenvolvimento pelo mundo (processo de catching up). Esse é um processo que precisa ser estimulado dentro do SPIn. 
Quadro 2 - Eixos Estratégicos 3 e 4: Proposta linhas de ações para o Sistema Pernambucano de Inovação (SPIn)

\begin{tabular}{|c|c|c|}
\hline EIXO ESTRATÉGICO E OBJETIVO & Dimensão & LiNHAS DE AÇÕES \\
\hline $\begin{array}{c}\text { Eixo Estratégico 3: } \\
\text { Aceleração da inovação nas } \\
\text { atividades econômicas } \\
\text { Objetivo: ampliar a geração de } \\
\text { valor e as competências inovativas. }\end{array}$ & $\begin{array}{c}\text { Intermediação } \\
\text { Interação entre } \\
\text { empresas, centros } \\
\text { de conhecimento } \\
\text { e demais agentes } \\
\text { do SRI }\end{array}$ & $\begin{array}{l}\text { - Estimular a criação de Núcleos de Inovação Tecnológica (NITs) } \\
\text { dentro das empresas públicas pernambucanas (Compesa, } \\
\text { Lafepe, Copergás, IPA) para gerir a PI produzida. } \\
\text { - Mapear as tecnologias do estado e produzir um "Radar } \\
\text { tecnológico" anual com dados de patentes ligados a tecnologias } \\
\text { do estado. } \\
\text { - Estimular o aprendizado tecnológico nas indústrias do estado } \\
\text { com a utilização de tecnologias não protegidas ou em domínio } \\
\text { público. } \\
\text { - Criar um Centro de Monitoramento Tecnológico estadual } \\
\text { para subsidiar as empresas e grupos de pesquisa quanto à } \\
\text { informação tecnológica. } \\
\text { - Mapear as tecnologias do estado e produzir um "Radar } \\
\text { tecnológico" anual com dados de patentes ligados a tecnologias } \\
\text { do estado. }\end{array}$ \\
\hline $\begin{array}{l}\text { Eixo Estratégico 4: } \\
\text { Cooperação e transferência } \\
\text { de conhecimento } \\
\text { Objetivo: ampliar a capacidade } \\
\text { e otimizar tempo, recursos e } \\
\text { resultados para inovação. }\end{array}$ & $\begin{array}{c}\text { Capacitação } \\
\text { e Gestão } \\
\text { empresarial }\end{array}$ & $\begin{array}{l}\text { - Promover rodadas de transferência de tecnologia no estado } \\
\text { em eventos de tecnologias. } \\
\text { - Criar junto ao IEL/Federação das Indústrias de Pernambuco } \\
\text { (FIEPE) unidade fixa de capacitação em PI para empresas e } \\
\text { indústrias do estado. } \\
\text { - Utilizar o Sebrae por meio do programa Sebratec para estimular } \\
\text { a proteção de tecnologias desenvolvidas por MPEs. } \\
\text { - Criar uma vitrine tecnológica com as patentes do estado (virtual } \\
\text { e impressa) e sua promoção em missões de negócios no Brasil } \\
\text { e no exterior. } \\
\text { - Utilizar o formato dos "Multiplicadores de PI" do INPI com a } \\
\text { APEX/Brasil para atuarem em diversas associações de empresas } \\
\text { e junto às MPEs com potencial de exportação do estado. } \\
\text { - Mapear as possíveis Indicações Geográficas (IGs) do estado } \\
\text { através da Agência de Desenvolvimento do Estado (ADdiper). }\end{array}$ \\
\hline
\end{tabular}

Fonte: Elaborado pelos autores deste artigo (2018)

No Quadro 3, são apresentadas linhas de ações para os Eixos Estratégicos 5 e 6 que congregam instituições dentro do SRI bastante delicadas e extremamente necessárias para o processo de inovação: financeiro e de governança. Para Cooke, Uranga e Etxebarria (1997), existem três pontos cruciais para o SRI de fato ser bem-sucedido: a capacidade financeira, de aprendizagem e a capacidade local de promover uma cultural de inovação. Por isso, as agências de fomento do estado de Pernambuco precisam estar atentas para a dinâmica do sistema de PI e contemplarem tais exigências em seus editais de subvenção econômica, por exemplo.

Por sua vez, Lima et al. (2007) relatam que a difusão e a inovação tecnológica acorrem quando há ambientes favoráveis e com uma cultura que esteja propícia a aglomerar profissionais estimulados a aprender e a trocar novas experiências. $\mathrm{O}$ autor enfatiza que, por meio de políticas públicas bem-sucedidas, ambientes que não possuem uma cultura inovadora podem ser favorecidos com ações estratégicas. Portanto, deve ser prioridade criar condições por meio de linhas de ações bem construídas e planejadas para que o SPIn consiga promover a interação entre os seus atores com uma gestão pública voltada para a inovação.

Para Mahroum e AlSaleh (2013), a mensagem mais importante no conceito de SI é de que os atores não podem, e não devem, inovar isoladamente e, portanto, a inovação é um processo coletivo e interativo. Portanto, as inovações são socialmente construídas e não correm de forma isolada (NIELSEN, 2010). 
Quadro 3 - Eixos Estratégicos 5 e 6: Proposta linhas de ações para o Sistema Pernambucano de Inovação (SPIn)

\begin{tabular}{|c|c|c|}
\hline EIXo ESTRATÉGICO E OBJETIVO & Dimensão & LiNHAS DE AÇÕES \\
\hline $\begin{array}{c}\text { Eixo Estratégico 5: } \\
\text { Ambiente favorável à inovação } \\
\text { Objetivo: aprimorar os } \\
\text { instrumentos e instituições para } \\
\text { ampliar a capacidade inovativa. }\end{array}$ & Financeira & $\begin{array}{l}\text { - Criar uma Política Estadual de Propriedade Intelectual. } \\
\text { - Propor aos editais de subvenção econômica das agências de } \\
\text { fomento do estado (Agefepe e Facepe) de novos produtos e } \\
\text { processos um depósito de pedido de patente sobre a tecnologia } \\
\text { desenvolvida. } \\
\text { - Prever na nova Lei de Inovação do Estado (em tramitação) } \\
\text { a PI como forma de contribuir e estimular o desenvolvimento } \\
\text { tecnológico do estado. } \\
\text { - Articular isenções e incentivos para as empresas do estado que } \\
\text { adquirem carta-patente. }\end{array}$ \\
\hline $\begin{array}{c}\text { Eixo Estratégico 6: } \\
\text { Governança e responsabilidade } \\
\text { Objetivo: calibrar a política } \\
\text { pública e despertar compromisso } \\
\text { na sociedade por CT\&I. }\end{array}$ & $\begin{array}{c}\text { Governança } \\
\text { Elaboração e } \\
\text { gestão de políticas }\end{array}$ & $\begin{array}{l}\text { - Criar um comitê de PI dentro da Secti e Fiepe com diversos } \\
\text { atores do SPIn. } \\
\text { - Agregar mais parceiros, como o INPI, no projeto "Caravana } \\
\text { da Inovação" pelo interior do estado de PE. } \\
\text { - Estimular uma pauta de discussão sobre PI junto à Assembleia } \\
\text { Legislativa do Estado (ALEPE). } \\
\text { - Aplicar em toda rede pública de ensino estadual, a } \\
\text { obrigatoriedade da disciplina de Empreendedorismo, Inovação } \\
\text { e PI. }\end{array}$ \\
\hline
\end{tabular}

Fonte: Elaborado pelos autores deste artigo (2018)

\section{Considerações Finais}

O foco deste trabalho foi apresentar um conjunto de propostas de disseminação do conhecimento da PI que pudessem ser aplicadas ao SPIn. Os resultados mostraram de forma diversificada as possibilidades de se impactar, a longo prazo, os diferentes atores do caso estudado, promovendo ações que pudessem propiciar um ambiente de troca de informações e de conhecimento da PI constante. A aplicação de boas práticas de políticas públicas voltadas para PI do Japão e da Coreia do Sul, por exemplo, propiciaram sugestões para serem adaptadas ao SPIn.

Como destacado por Cooke, Uranga e Etxebarria (1997), a capacidade de aprendizagem institucional é um dos pontos cruciais para que o SRI consiga prosperar de alguma forma. Segundo o autor, apresentar uma homogeneidade entre o conhecimento aprendido pelos atores e possuir uma coesão social dentro das instituições facilitam o processo de inovação.

Segundo Ogiya (2016), anualmente o governo japonês define a estratégia de PI que será seguida no ano posterior com todos os envolvidos: Setor Privado (empresas, indústria, associações, universidades etc.) e o Governo (Gabinete Oficial, Ministérios da Justiça, da Economia, da Indústria e Serviços, da Agricultura, da Educação e da CT\&I). Enfim, todos participam e alinham as estratégias que serão delineadas e seguidas por todos.

Criar uma disseminação sobre o conhecimento da PI entre todos os atores do Sistema Pernambucano de Inovação deve fazer parte de todo documento estratégico da política de ciência, tecnologia e inovação nacional e estadual para que se consiga competir de igual para igual num mercado globalizado. A promoção de uma cultura é criada desde a base da educação até os grandes centros de P\&D por meio de cursos, minicursos, palestras, seminários, oficinas de busca e redação de patentes e diversas outras interações que promovam a discussão e o uso estratégico dessa ferramenta. É importante propagar os benefícios e as vantagens desse sistema 
que pretende retribuir ao criador o esforço de tempo e de investimentos gastos na criação de novas tecnologias.

A criação de políticas públicas voltadas para inovação com a utilização estratégica da PI pode resultar em um crescimento social e econômico para o SPIn e para o todo o entorno. Percebe-se, portanto, a necessidade de disseminar o conhecimento da propriedade industrial nas universidades e escolas, nas instituições de CT\&I, nas empresas públicas, nas agências de fomento e nas demais organizações do Estado de Pernambuco para, assim, por meio da interação e da cooperação entre os atores, seja possível fomentar o sistema local de inovação.

\section{Referências}

AMORIM-BORHER, M. B. O management do intangível. Revista Propriedade e Ética, [S.l.], ano 1, n. 2, mar./abr., 2008.

AMORIM-BORHER, M. B. et al. Ensino e pesquisa em propriedade intelectual no Brasil. Revista Brasileira de Inovação, [S.l.], v. 6,n. 2, p. 281-310, jul./dez., 2007.

AUTIO, E. Evaluation of RTD in regional systems of innovation. European Planning Studies, [S.l.], v. 6, n. 2, p. 131-140, 1998.

BARBOSA, D. B. Uma introdução à propriedade intelectual - Patentes. Rio de Janeiro: Ed. Lumen Juris, 2010.

CASSIOLATO, J. E.; ZUCOLOTO, G.F. Desenvolvimento tecnológico por empresas estrangeiras no Brasil e na Coreia do Sul. Revista da Economia Contemporânea, Rio de Janeiro, v. 18, n. 2, 2014.

CHANG, H. J. Kicking away the ladder: development strategy in historical perspective. Londres: Anthem Press, 2002.

COOKE, P.; URANGA, M. G.; ETXEBARRIA, G. Regional systems of innovation: an evolutionary perspective. Environment and Planning, [S.I.], v. 30, p. 1.563-1.584, 1997.

COOKE, P. Regional innovation systems, clusters, and the knowledge economy. Industrial and Corporate Change, Oxford, v. 10, n. 4, p. 945-974, Aug. 2001.

COOKE, P. Regionally asymmetric knowledge capabilities and open innovation: Exploring 'Globalisation 2' - A new model of industry organisation. Research Policy, [S.I.], v. 34, n. 8, p. 1.128-1.149, 2005.

DOLOREUX, D. What we should know about regional systems of innovation. Technology in Society, [S.I.], v. 24, n. 3, p. 243-263, 2002.

DOLOREUX, D.; PARTO, S. Regional innovation systems: current discourse and unresolved issues. Technology in Society, [S.l.], v. 27, p. 133-153, 2005.

DRAHOS, P. Global property rights in information: the story of TRIPS at the GATT Prometheus, [S.l.], v. 13, n. 1, 1995.

DUBEUX, R. R. Inovação no Brasil e na Coréia do Sul. Curitiba: Juruá, 2010. 
ECT\&I-PE - ESTRATÉGIA DE CIÊNCIA, TECNOLOGIA E INOVAÇÃO PARA PERNAMBUCO. 2017-2022. [2017]. Disponível em: <http://www.secti.pe.gov.br/wp-content/uploads/2017/04/ Estrategia\%20de\%20Ciencia\%20Tecnologia\%20e\%20Inovacao\%20-\%20FINAL.pdf > . Acesso em: 20 mar. 2018.

FREEMAN, C. Technology policy and economic performance: lessons from Japan. London: Frances Pinter, 1987.

GANDELMAN, M. Poder e Conhecimento na Economia Global. Rio de janeiro: Editora Civilização Brasileira, 2004.

GUIMARÃES, A. C. O papel do Instituto Nacional de Propriedade Industrial no processo de capacitação em propriedade intelectual no Brasil de 2004 a 2011. Rio de Janeiro: Instituto Nacional de Propriedade Industrial, 2013.

INSTITUTO BRASILEIRO DE GEOGRAFIA E ESTATÍSTICA - IBGE. [2016]. Disponível em: <https://www.ibge.gov.br/estatisticas-novoportal/economicas/>. Acesso em: 19 jun. 2018.

INSTITUTO NACIONAL DE PROPRIEDADE INDUSTRIAL (IMPI). [2016]. Disponível em: < http:// www.inpi.gov.br/sobre/estatisticas>. Acesso em: 18 jun. 2018.

KIM, L. Da imitação à inovação: a dinâmica do aprendizado tecnológico da Coréia. Campinas: Ed. da Unicamp, 2005.

LIMA, M. C.; FERREIRA, J.; FERNANDES, A. C. A Dimensão Regional do Sistema Brasileiro de Inovação. Rio Janeiro: Nota Técnica. CGGE, 2007.

LUNDVALL, B. A. National innovation systems: towards a theory of innovation and interactive learning. London: Pinter, 1992.

MAHROUM, S.; ALSALEH, Y. Towards a functional framework for measuring national innovation efficacy. Technovation, [S.l.], v. 33, n. 10, p. 320-332, 2013.

MENDES, L. M.; BOHER, B. A. O ensino da propriedade intelectual: mapeando as academias da rede global. Revista Brasileira de Inovação, Campinas, SP, v. 11, n. 2, p. 399-432, 2012.

NELSON, R. The Oxford Handbook of Innovation. Oxford Business Press, Londres, p. 209-239, 2005 .

NELSON, R. R. (Ed.). National Innovation Systems: a Comparative Study. New York: Oxford University Press, 1993.

NIELSEN, H. Universities in Regional Systems of Innovation: has the Rural Research Center in Höfn Enhanced the Interaction between the University and Local Actors? Master's thesis. CIRCLE, Lund University, 2010.

MINISTÉRIO DA CIÊNCIA, TECNOLOGIA, INOVAÇÕES E COMUNICAÇÕES (MCTI). [2014]. Disponível em: <https:/www.mctic.gov.br/mctic/opencms/indicadores/detalhe/recursos_aplicados/ RecursosAplicados-CeT.html>. Acesso em: 18 jun. 2018.

OGIYA, T. Intellectual Property Basic Atc and IP Human Resource Development. JPO, 2016. ORGANIZAÇÃO MUNDIAL DA PROPRIEDADE INTELECTUAL - OMPI. [2017a]. Disponível em: <https://www3.wipo.int/ipstats/ipslinechart> Acesso em: 19 jun. 2018. 
ORGANIZAÇÃO MUNDIAL DA PROPRIEDADE INTELECTUAL (OMPI). [2017b]. Disponível: <www.wipo.int/edocs/pubdocs/en/intproperty/.../wipo_pub_944_2011.pdf>. Acesso em: 15 jun. 2018.

SECTI-PE. Boletim de Indicadores de Ciência, Tecnologia e Inovação. Atividades de Patenteamento no Brasil e no Estado de Pernambuco. [2015]. Disponível em: <http://www.secti. pe.gov.br/wp-content/uploads/2016/03/Boletim_Patentes.pdf > . Acesso em: 20 jun. 2018.

SHERWOOD, R. M. Propriedade Intelectual e Desenvolvimento Econômico. São Paulo, Ed. Edusp, 1992.

SOUSA JÚNIOR, C. C. de. O Sistema Regional de Inovação do estado de Minas Gerais: uma análise a partir de suas organizações e interações. 2014. 155p. Dissertação (Mestrado) Departamento de Fisiologia e Biofísica, Universidade Federal de Minas Gerais, Belo Horizonte, Minas Gerais, 2014.

SUN, J. C.; BAEZ, B. Intellectual Property in the information Age: Knowledge as Commodity and Its Legal Implication for Higher Education. ASHE Hiher Education Report, [S.I.],v. 34, n. 4, 2009.

TAKAGI, Y.; ALLMAN, L.; SINJELA, M. A. Teaching of Intellectual Property. New York, Cambridge University Press, 2008.

\section{Sobre os autores}

\section{André Marques Cavalcanti}

E-mail: andremarques2008@gmail.com

Coordenador e Professor do Programa de Mestrado Profissional em Propriedade Intelectual e Transferência de Tecnologia para Inovação (PROFNIT) ponto focal UFPE.

Endereço profissional: Av. dos Economistas, s/n, Centro de Ciências Sociais Aplicadas, Departamento de Ciências Administrativas, UFPE, Cidade Universitária, Recife, PE. CEP: 50740-590.

\section{Eduardo Andrade Bemfica}

E-mail: eduardobemfica@hotmail.com

Mestrado Profissional em Propriedade Intelectual e Transferência de Tecnologia para a Inovação (PROFNIT).

Endereço profissional: Av. Prof. Moraes Rego, n. 1.235, SL 108 (INPI), Edifício POSITIVA, Diretoria de Inovação, UFPE, Cidade Universitária, Recife, PE. CEP: 50670-901. 